\title{
High-Throughput Assessment and Genetic Investigation of Vegetative Compatibility in Verticillium dahliae
}

\author{
Ioannis A. Papaioannou and Milton A. Typas \\ Department of Genetics and Biotechnology, Faculty of Biology, National and Kapodistrian University of Athens, Panepistimiopolis 15701, Athens, \\ Greece
}

\section{Keywords}

complementation tests, heterokaryon incompatibility, nit mutants, population biology, VCGs

\section{Correspondence \\ M. A. Typas, Department of Genetics and Biotechnology, Faculty of Biology, National and Kapodistrian University of Athens, Athens, Greece. \\ E-mail: matypas@biol.uoa.gr}

Received: May 22, 2014; accepted: October 5, 2014.

doi: 10.1111/jph.12345

\begin{abstract}
Classification of isolates into vegetative compatibility groups (VCGs) using nitrate-non-utilizing (nit) mutants has been widely used for the characterization of Verticillium dahliae populations. However, certain methodological limitations prevent its application on a large scale. Furthermore, systematic investigations into the genetics underlying complementation tests between nit mutants of fungal isolates (i.e. heterokaryon formation) are lacking for Verticillium species. In this work, a diverse collection of 27 $V$. dahliae isolates - including representatives of all VCGs, both mating types, and heterokaryon self-incompatible isolates - was employed for the development and optimization of (i) a protocol for the rapid generation of nit mutants of $V$. dahliae isolates using UV-irradiation and (ii) a reproducible high-throughput procedure for complementation tests between nit mutants in liquid cultures using 96-well microplates. The genetic analysis of selected heterokaryons demonstrated that the frequently encountered 'weak' cross-reactions between VCGs and their subgroups can be actually heterokaryotic, implying the absence of strict genetic barriers between VCGs. In conclusion, we provide in this work an optimized method for the high-throughput VCG assignment of $V$. dahliae populations and a genetic analysis of heterokaryons that may have serious implications for the interpretation of VCG classification data. These advancements in the available methodology and the genetic background of vegetative compatibility grouping may contribute to a better understanding of the population biology of $V$. dahliae and possibly other mitosporic fungi.
\end{abstract}

\section{Introduction}

Verticillium dahliae is a soilborne phytopathogenic ascomycete that causes vascular wilt in more than 400 plants. The limited host specialization of the fungus, its vascular habitat, the longevity of its resting structures and the shortage of resistant host plant varieties have hindered control of the notorious Verticillium wilt disease (Klosterman et al. 2009). A thorough understanding of the genetic diversity and the relationships within and between $V$. dahliae populations is predicted to facilitate effective disease management (López-Escudero and Mercado-Blanco 2011).
Although mating-type genes have been described in $V$. dahliae (Usami et al. 2009), and population genetics analyses have revealed some putative signs of sex (Atallah et al. 2010; Inderbitzin et al. 2011), a sexual stage of the fungus has never been found so far, and $V$. dahliae populations are considered to be clonally propagating for the most part (Klosterman et al. 2009; Gurung et al. 2014). Apart from the accumulation of mutations (Anderson and Kohn 1995) and chromosomal rearrangements (de Jonge et al. 2013), genetic variation may be generated with restricted exchange and reassortment of genetic information through heterokaryosis and the subsequent parasexual cycle (Puhalla and Mayfield 1974; Typas 
and Heale 1978). However, heterokaryon formation is often prevented by vegetative incompatibility systems (Typas and Heale 1976), similar to what is observed in most fungi (Leslie 1993). Different methods have been employed for the classification of $V$. dahliae isolates according to their compatibility behaviour (Typas and Heale 1976; Puhalla and Hummel 1983; Joaquim and Rowe 1990). Among them, complementation tests of nitrate-non-utilizing (nit) mutants have been widely adopted in the last two decades for the characterization of $V$. dahliae populations. According to this method, $V$. dahliae isolates are assigned to the same vegetative compatibility group (VCG) if viable heterokaryons with prototrophic-like growth are formed when complementary nit mutants of the isolates are confronted on minimal medium (Joaquim and Rowe 1990). Five main VCGs are generally recognized today for $V$. dahliae populations, that is VCGs 1-4 and 6, with each of VCGs 1, 2 and 4 being subdivided into $A$ and $B$ subgroups, depending on the extent of heterokaryon formation; VCGs 3 and 6 are only rarely encountered (Katan 2000; Bhat et al. 2003; Jiménez-Díaz et al. 2006; Papaioannou et al. 2013b).

Extensive research in model fungi, such as Neurospora crassa and Podospora anserina, has revealed that incompatibility is governed by a set of heterokaryon (het) or vegetative incompatibility (vic) loci, with hyphal compartmentalization and programmed cell death being triggered in any heterokaryon that contains nuclei with different alleles at any one of these loci (Hutchison and Glass 2012). A similar genetic control of incompatibility is predicted for other fungi, although the relevant gene repertoire is still unknown for almost all of them. Based on this assumption, it is inferred that all members of a VCG of an asexual fungus are identical at all het loci, which may probably result from common descent. Thus, each VCG can be often regarded as a clonal lineage that is isolated from other VCGs. Members of distinct VCGs have been traditionally hypothesized to differ in several physiological and pathogenicity-related characteristics, rendering VCG analysis a convenient and informative tool for the characterization of fungal populations (Leslie 1993; Katan 2000).

A number of recent studies have revealed a previously unexpected complexity regarding molecular relationships within $V$. dahliae VCGs and between their subgroups, by employing different molecular tools (Collado-Romero et al. 2006, 2008; Martin 2010; Papaioannou et al. 2013a,b). Remarkably, main VCGs 2 and 4 were overall demonstrated to comprise molecularly distinct subgroups, with each of them being more closely related to a subgroup of the other VCG than its 'sister' subgroup (i.e. a VCG $2 \mathrm{~A}-4 \mathrm{~B}$ and 2B-4A pattern of relatedness was observed), and VCG 2B was shown to be highly heterogeneous and polyphyletic (Collado-Romero et al. 2008; Papaioannou et al. 2013a,b; Jiménez-Gasco et al. 2014). Despite these complications, however, and although elaborate molecular methods and tools for the characterization of fungal populations at a high resolution are increasingly available today (Martin 2010; Davey et al. 2011; Papaioannou et al. 2013a), traditional VCG analysis is still important for the study of $V$. dahliae populations. A relationship between certain VCGs and the taxonomic position of the original host of $V$. dahliae isolates has been recorded, for example for VCGs 1 and 2 with cotton and VCG 4 with solanaceous plants (Joaquim and Rowe 1990; Daayf et al. 1995). Furthermore, certain VCGs or subgroups have been correlated in several cases with virulence on specific hosts such as tomato, potato, cotton, olive, eggplant, sweet pepper and artichoke (e.g. Joaquim and Rowe 1991; Strausbaugh et al. 1992; Daayf et al. 1995; Korolev et al. 2000; Tsror (Lahkim) et al. 2001; Bhat et al. 2003; Collins et al. 2005; Jiménez-Díaz et al. 2006). In addition, significant differences in morphology and physiology have been reported between different VCGs (Korolev et al. 2000; Zeise and von Tiedemann 2001; Papaioannou et al. 2013b). The phytopathological significance of VCG analyses for $V$. dahliae populations is reflected in the unabated frequency of relevant reports in the literature (some recent examples include Dung et al. 2013; El-Bebany et al. 2013; Iglesias-Garcia et al. 2013; Papaioannou et al. 2013b).

Although VCG classification using nit mutants proved sufficiently convenient to allow research-level investigations in $V$. dahliae, it appears impractical for application on a larger scale (Leslie 1993). Microplatebased complementation tests on MM agar have been previously used with Fusarium species (Klittich and Leslie 1988), but an equivalent high-throughput method has never been reported for Verticillium. Such methodological limitations hinder VCG classification of extended populations of the fungus, which may be essential for the determination of significant correlations between the VCG system and the fungal phenotype, as well as for large-scale molecular analyses of relationships on the VCG level. Furthermore, a significant variability in the extent of prototrophic growth has generally been observed among different pairings of nit mutants, ranging from reactions that resemble wild-type prototrophic growth ('strong' reactions) to less vigorous interactions with sparse aerial hyphae and slow growth rates ('weak' reactions); marginal 
reactions have also been detected, usually characterized by dots of microsclerotia along the contact line of the confronted nit mutants (Joaquim and Rowe 1990; Daayf et al. 1995; Korolev et al. 2000; Göre 2009; Papaioannou et al. 2013b). The traditional VCG classification system for $V$. dahliae depends mostly on 'strong' and reproducible reactions for the assignment of isolates into VCGs, although a detailed genetic comparison of the different types of interactions has never been reported. This analysis may advance our understanding of heterokaryon incompatibility in $V$. dahliae and contribute towards reconsidering the 'traditional' way VCG classification data is interpreted.

The aims of this study, therefore, were (i) to expedite VCG classification of $V$. dahliae isolates by applying UV-mutagenesis for the rapid generation of nit mutants and developing a novel method for their consistent and high-throughput complementation testing and (ii) to compare genetically for the first time the two types of complementation (i.e. 'strong' and 'weak' reactions) that are frequently observed during VCG assignment tests.

\section{Materials and Methods}

Fungal isolates, growth media and culture conditions

A total of $27 \mathrm{~V}$. dahliae isolates from various hosts and geographic origins were employed in this study (Table 1). Members of all VCGs - as well as three heterokaryon self-incompatible (HSI) isolates - and representatives of both mating types of this species were included in the collection. Potato dextrose agar (PDA) and Czapek-Dox agar (Typas and Heale 1976) were used as the complete and minimal medium (MM), respectively, except for the characterization of nit mutants, which was performed on minimal media with different nitrogen sources (Correll et al. 1987). Monoconidial cultures were obtained from all isolates and were stored as conidial suspensions (at concentrations of at least $10^{6}$ conidia $/ \mathrm{ml}$ ) in potato dextrose broth (PDB) with $20 \%$ glycerol, at $-80^{\circ} \mathrm{C}$. Prior to each experiment, active cultures of the isolates were obtained directly from glycerol stocks by plating aliquots on PDA plates and incubating them for 1 week at $24^{\circ} \mathrm{C}$, in the dark.

\section{Production and characterization of nit mutants}

Nit mutants of $V$. dahliae isolates were obtained either by the standard procedure involving selection of resistant sectors of agar inocula (Joaquim and Rowe 1990) on the chlorate-amended medium WAC (Korolev and
Table 1 Verticillium dahliae isolates used in this study with code names, VCGs, original hosts and geographic origins (sources)

\begin{tabular}{|c|c|c|c|}
\hline Isolate codes & $\mathrm{VCG}^{\mathrm{a}}$ & Host & Origin (source) \\
\hline T9 [A] & $1 \mathrm{~A}(\mathrm{a})$ & Cotton & USA, CA (1) \\
\hline V44 [B] & $1 \mathrm{~A}(\mathrm{a})$ & Cotton & USA, TX (1) \\
\hline V607I (R04) [C] & 1B (b) & Green ash & USA, MN (2) \\
\hline V661I (328v-1) [D] & $1 \mathrm{~B} \mathrm{HSI}{ }^{\mathrm{C}}(\mathrm{b})$ & Cotton & Greece (2) \\
\hline $\mathrm{PH}[\mathrm{E}]$ & $2 \mathrm{~A}(\mathrm{a})$ & Pistachio & USA, CA (1) \\
\hline V320I [F] & $2 \mathrm{~A}(\mathrm{~b})$ & Cotton & USA, CA (2) \\
\hline $115[\mathrm{G}]$ & $2 B(a)$ & Cotton & Syria (1) \\
\hline Ls.17 [H] & $2 B(c)$ & Lettuce & USA, CA (3) \\
\hline V16 [I] & $2 B(d)$ & Potato & Germany (4) \\
\hline Cf. $38^{d}[J]$ & $2 B(e, f)$ & Chili pepper & USA, CA (3) \\
\hline V702I [K] & 2Ba (g) & Artichoke & Spain (2) \\
\hline $530-1[\mathrm{~L}]$ & $2 \mathrm{~B}, 2 \mathrm{~A}(\mathrm{~h})$ & Pepper & Greece (5) \\
\hline $25 \mathrm{~V}$ (SS4) [M] & $2 A B, 1,4 A(i)$ & Cotton & USA (6) \\
\hline $70-21[N]$ & $3(j)$ & Pepper & USA, AZ (1) \\
\hline PCW [O] & 3 (a) & Pepper & USA, CA (1) \\
\hline $\mathrm{BB}[\mathrm{P}]$ & $4 \mathrm{~A}(\mathrm{a})$ & Potato & USA, ID (1) \\
\hline V830 [Q] & $4 \mathrm{~A}(\mathrm{~d})$ & Potato & USA, OH (4) \\
\hline Dvd-E6 [R] & $4 \mathrm{~A}(\mathrm{k})$ & Eggplant & Canada (7) \\
\hline S39 [S] & $4 \mathrm{~B}(\mathrm{a})$ & Soil & USA, OH (1) \\
\hline V684I [T] & $4 \mathrm{~B}(\mathrm{~g})$ & Artichoke & Spain (2) \\
\hline Ca.83 [U] & $6(\mathrm{e})$ & Bell pepper & USA, CA (3) \\
\hline Ca. $146^{\mathrm{e}}[\mathrm{V}]$ & $6(e)$ & Bell pepper & USA, CA (3) \\
\hline Ca.148 [W] & $6(e)$ & Bell pepper & USA, CA (3) \\
\hline Cf. $162^{d}[X]$ & $\mathrm{HSI}^{f}(e, f)$ & Chili pepper & USA, CA (3) \\
\hline $\mathrm{V} 13[\mathrm{Y}]$ & $\mathrm{HSI}^{f}(\mathrm{~d})$ & Cotton & Spain (4) \\
\hline $\mathrm{CA} 26^{\mathrm{e}}[\mathrm{Z}]$ & - & Cabbage & Japan (8) \\
\hline TO- $0^{\mathrm{e}}$ & - & Tomato & Japan (8) \\
\hline
\end{tabular}

aData on 'bridging' behaviour (i.e. complementation to varying degrees with tester isolates of more than one VCG subgroups) were available for several $V$. dahliae isolates that were included in this study; these secondary VCG interactions are provided after commas. The reference of the study reporting the VCG assignment of each isolate is provided in brackets, as follows: a, Joaquim and Rowe (1990); b, Collado-Romero et al. (2006); c, Papaioannou and Typas, unpublished data; d, Zeise and von Tiedemann (2001); e, Bhat et al. (2003); f, Papaioannou et al. (2014); g, Jiménez-Díaz et al. (2006); h, Papaioannou et al. (2013b); i, Elena and Paplomatas (1998); j, Joaquim and Rowe (1991); k, Dobinson et al. (1998).

${ }^{\mathrm{b}}$ Source: $1=\mathrm{R}$. Rowe, OARDC, The Ohio State University, USA; $2=\mathrm{M}$. Jiménez-Gasco, The Pennsylvania State University, USA; 3 = K. Subbarao, University of California, Davis, USA; 4 = A. von Tiedemann, University of Göttingen, Germany; $5=$ E. Ligoxigakis, Plant Protection Institute, N.AG.RE.F., Greece; 6 = E. Paplomatas, Agriculture University of Athens, Greece; $7=$ K. Dobinson, University of Western Ontario, Canada \& Agriculture and Agri-Food, Canada; $8=$ T. Usami, Chiba University, Japan.

${ }^{\mathrm{C}}$ This isolate was originally reported as heterokaryon self-incompatible (HSI), but vegetatively compatible with isolates of VCG 1B (Collado-Romero et al. 2006).

${ }^{\mathrm{d}}$ The VCG classification of these isolates, which were originally assigned to VCG 6 (Bhat et al. 2003), was re-examined by Papaioannou et al. (2014), and the revised classification is provided here.

${ }^{\text {e }}$ Reported to bear the MAT1-1-1 mating-type idiomorph (Usami et al. 2009), similar to isolate Ca.146 (Papaioannou et al. 2013b). Representative tester isolates of all VCGs that are included in this work have been previously assigned to the MAT1-2-1 idiomorphic type (Papaioannou et al. 2013b).

${ }^{\mathrm{f}} \mathrm{HSI}$, heterokaryon self-incompatible. 
Katan 1997) or by exposing conidia to ultraviolet (UV) irradiation (Typas and Heale 1976). In the latter method, approximately $10^{5}$ conidia of each isolate were spread on WAC medium plates prior to exposure at $20 \mathrm{~cm}$ from a germicidal UV-C lamp emitting 95\% of the radiation at $254 \mathrm{~nm}$. At this distance, the radiation intensity was estimated at approximately $110 \mu \mathrm{W} / \mathrm{cm}^{2}$, and the UV dosage was adjusted to a $5 \%$ survival rate by exposing conidia for 20 s. Plates were scored for the emergence of chlorate-resistant colonies up to 10 days post-UV-irradiation. Phenotype identification of all nit mutants produced by either method was performed by growth assays on MM with different nitrogen sources (Correll et al. 1987). The reversion rates of nit mutants were calculated by spreading dense conidial suspensions of each isolate on MM and viable counting on PDA, after appropriate serial dilutions.

\section{Complementation tests and heterokaryon analysis}

Complementation tests between nit mutants on solid MM were performed according to standard procedures (Joaquim and Rowe 1990), except that plates were scored for prototrophic growth up to 5 weeks after inoculation. All pairings were performed in three independent repetitions. Heterokaryon analysis was also carried out according to previously described methods (Typas and Heale 1976; Puhalla and Spieth 1983). Ninety-six-well microplates were routinely used for complementation tests between nit mutants in liquid MM, and several methods of inoculation were tested in a preliminary investigation. The optimal procedure that was selected for all subsequent experiments involved (i) mixing 25- $\mu$ l aliquots of conidial suspensions (with concentrations ranging from $10^{5}$ to $10^{6}$ conidia $/ \mathrm{ml}$ ) of two isolates per pairing in each well of the microplate, (ii) addition of liquid MM to a final volume of $100 \mu \mathrm{l}$ in each well and (iii) static incubation of plates at $24^{\circ} \mathrm{C}$, in the dark, for 10 days. Following incubation, compatibility of nit mutants in each well was assessed by recording the amount of prototrophic mycelial growth (an example is provided in Fig. 1). At least two independent nit mutants of each isolate were tested, and all pairings were performed in four to eight repetitions each. To rule out the possibility of reversion, the mycelial mats of selected pairings were transferred onto MM agar plates, and their conidia were checked for nit phenotype or prototrophy. To test the congruence between the results of the new method and the traditional complementation tests of nit mutants on solid MM, all pairings were repeated on MM agar, in three repetitions each, according to standard procedures (Joaquim and Rowe 1990), and results were recorded up to 5 weeks after inoculation. When sterile cellophane sheets were interposed between the inocula of selected pairings, no growth was observed, suggesting that complementation was not due to cross-feeding (syntrophism).

\section{Results}

Rapid production of nit mutants with UV-mutagenesis

A total of 785 chlorate-resistant mutants (Table S1) were produced from the $27 \mathrm{~V}$. dahliae isolates of all

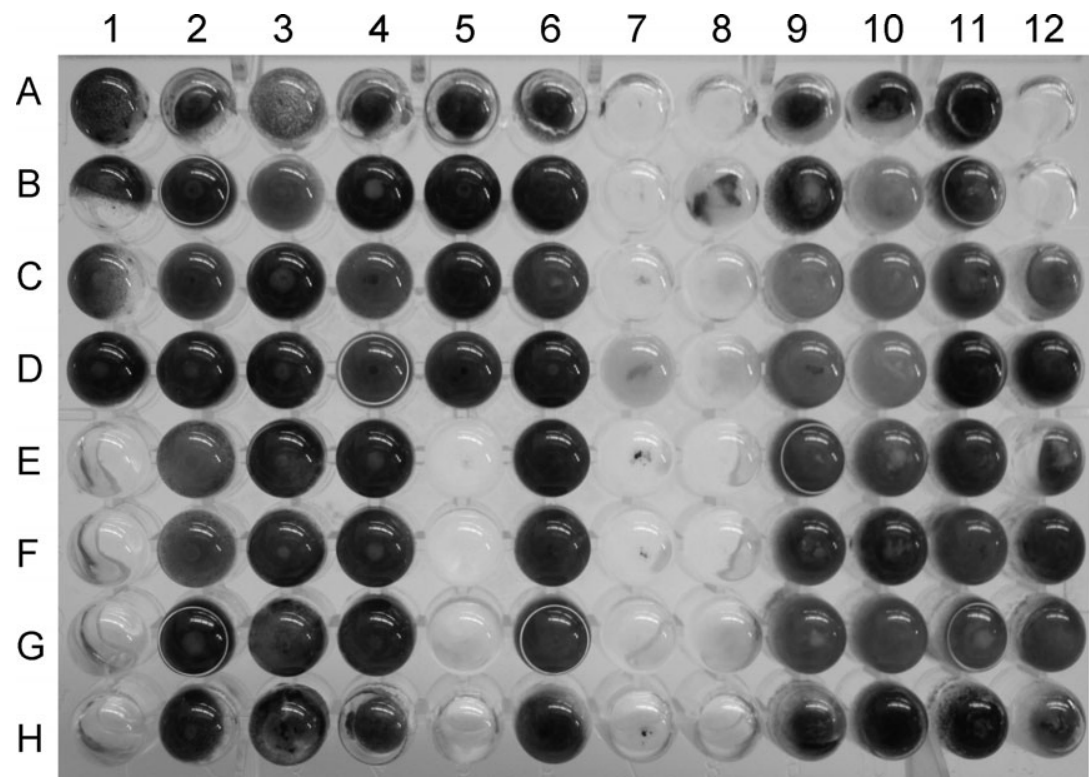

Fig. 1 Example of high-throughput complementation testing of several nit mutants of selected Verticillium dahliae isolates on a 96well microplate. Liquid MM (100 $\mu$ l), inoculated with mixed conidial suspensions of different pairs of nit mutants, is contained in each well, and the microplate has been incubated at $24^{\circ} \mathrm{C}$, in the dark, for 10 days. Considerable mycelial growth in a well, usually accompanied by significant pigment production, denotes complementation between the tested mutants in that well. 
VCGs (Table 1) with the agar plug sectoring and UVirradiation methods. Remarkably, UV-treatment resulted in high frequency of chlorate-resistant colonies, reaching an average of approximately $10^{-2}$, at a $5 \%$ survival rate, 10 days after UV-irradiation. Almost half of all chlorate-resistant colonies produced by both methods $(45.4 \%$ in total) were classified as stable nit mutants (i.e. no reversion to prototrophy was observed after at least three subculturings on MM). Both methods were equally successful for the generation of nitl (51.2 and 53.1\% of spontaneous and UVinduced mutants, respectively) and nitM (48.8 and $46.9 \%$, respectively) mutant classes. Although some variability in the mutants' growth characteristics was observed on nitrite, no actual nit 3 mutants were recovered, as intra-isolate complementation tests of all 'suspected' mutants allowed their assignment to the nitl class. Independent nitM mutants of some isolates could be assigned to three different complementation groups, which generally complemented each other more vigorously than complementation in intra-isolate pairings between nitl and nitM mutants (data not shown).

A significant proportion of chlorate-resistant colonies produced by both methods $(47.1 \%$ in total) were able to grow on MM with nitrate as the nitrogen source, after one to three subculturings on MM. Furthermore, $5.5 \%$ of all chlorate-resistant colonies exhibited wild-type growth characteristics on nitrate and nitrite but were unable to grow on hypoxanthine.

Table 2 Results from the new method for complementation testing of nit mutants produced from Verticillium dahliae isolates of all VCGs, in all possible combinations within and among VCG subgroups, in liquid MM microcultures ${ }^{a, b}$

\begin{tabular}{|c|c|c|c|c|c|c|c|c|c|c|c|c|c|c|c|c|c|c|c|c|c|c|c|c|c|c|}
\hline \multirow{2}{*}{$\begin{array}{l}\mathrm{VCG}^{\mathrm{C}} \\
\text { Isolate } \\
\text { code }\end{array}$} & \multicolumn{2}{|c|}{$1 \mathrm{~A}$} & \multicolumn{2}{|c|}{$1 \mathrm{~B}$} & \multicolumn{2}{|l|}{$2 \mathrm{~A}$} & \multicolumn{4}{|l|}{$2 B$} & \multirow{2}{*}{$\begin{array}{l}2 \mathrm{Ba} \\
\mathrm{K}\end{array}$} & \multicolumn{2}{|l|}{$2 A B$} & \multicolumn{2}{|l|}{3} & \multicolumn{3}{|l|}{$4 \mathrm{~A}$} & \multicolumn{2}{|l|}{$4 \mathrm{~B}$} & \multicolumn{3}{|l|}{6} & \multicolumn{2}{|c|}{$\mathrm{HSI}$} & \multirow{2}{*}{$\begin{array}{l}- \\
\text { Z }\end{array}$} \\
\hline & A & B & C & $D^{d}$ & $\mathrm{E}$ & $\mathrm{F}$ & G & $\mathrm{H}$ & 1 & J & & L & $M$ & $\mathrm{~N}$ & 0 & $P$ & Q & $\mathrm{R}$ & S & $\mathrm{T}$ & U & V & W & $x$ & Y & \\
\hline A & + & + & - & - & - & - & - & - & - & - & - & - & - & - & - & - & - & - & - & - & - & - & - & - & - & - \\
\hline B & + & + & - & - & - & - & - & - & - & - & - & - & - & - & - & - & - & - & - & - & - & - & - & - & - & - \\
\hline C & - & - & - & - & - & - & - & - & - & - & - & - & - & - & - & - & - & - & - & - & - & - & - & - & - & - \\
\hline D & - & - & - & - & - & - & - & - & - & - & - & - & - & - & - & - & - & - & - & - & - & - & - & - & - & - \\
\hline $\mathrm{E}$ & - & - & - & - & + & + & $+1-$ & + & $+1-$ & - & - & - & + & - & - & - & - & - & - & - & - & - & - & - & - & - \\
\hline$F$ & - & - & - & - & + & + & - & $+1-$ & + & - & $+1-$ & $+1-$ & $+1-$ & $+1-$ & - & $+1-$ & - & - & - & + & - & - & - & - & - & $+1-$ \\
\hline G & - & - & - & - & $+1-$ & - & + & + & - & $+1-$ & + & + & - & - & - & - & - & - & $+1-$ & - & - & - & - & - & - & $+1-$ \\
\hline $\mathrm{H}$ & - & - & - & - & + & $+1-$ & + & + & - & + & + & + & - & - & - & $+1-$ & - & - & - & - & - & - & - & - & - & $+1-$ \\
\hline I & - & - & - & - & $+1-$ & + & - & - & $+1-$ & - & - & - & - & - & - & - & - & - & - & - & - & - & - & - & - & - \\
\hline J & - & - & - & - & - & - & $+1-$ & + & - & + & + & - & - & - & - & - & - & - & - & - & - & - & - & - & - & - \\
\hline K & - & - & - & - & - & $+1-$ & + & + & - & + & + & + & - & - & - & - & - & - & - & - & - & - & - & - & - & - \\
\hline $\mathrm{L}$ & - & - & - & - & - & $+1-$ & + & + & - & - & + & $+1-$ & - & - & - & - & - & - & $+1-$ & - & - & - & - & - & - & - \\
\hline M & - & - & - & - & + & $+1-$ & - & - & - & - & - & - & $+1-$ & - & - & - & - & - & - & - & - & - & - & - & - & - \\
\hline N & - & - & - & - & - & $+1-$ & - & - & - & - & - & - & - & + & + & - & - & - & - & - & - & - & - & - & - & - \\
\hline 0 & - & - & - & - & - & - & - & - & - & - & - & - & - & + & + & $+1-$ & - & - & $+1-$ & - & - & - & - & - & - & - \\
\hline$P$ & - & - & - & - & - & $+1-$ & - & $+1-$ & - & - & - & - & - & - & $+1-$ & + & - & - & $+1-$ & $+1-$ & - & - & - & - & - & - \\
\hline Q & - & - & - & - & - & - & - & - & - & - & - & - & - & - & - & - & - & - & - & - & - & - & - & - & - & - \\
\hline $\mathrm{R}$ & - & - & - & - & - & - & - & - & - & - & - & - & - & - & - & - & - & - & - & - & - & - & - & - & - & - \\
\hline$S$ & - & - & - & - & - & - & $+1-$ & - & - & - & - & $+1-$ & - & - & $+1-$ & $+1-$ & - & - & + & + & - & - & - & - & - & - \\
\hline $\mathrm{T}$ & - & - & - & - & - & + & - & - & - & - & - & - & - & - & - & $+1-$ & - & - & + & + & - & - & - & - & - & - \\
\hline U & - & - & - & - & - & - & - & - & - & - & - & - & - & - & - & - & - & - & - & - & + & + & + & - & - & - \\
\hline V & - & - & - & - & - & - & - & - & - & - & - & - & - & - & - & - & - & - & - & - & + & + & + & - & - & - \\
\hline W & - & - & - & - & - & - & - & - & - & - & - & - & - & - & - & - & - & - & - & - & + & + & + & - & - & - \\
\hline$x$ & - & - & - & - & - & - & - & - & - & - & - & - & - & - & - & - & - & - & - & - & - & - & - & - & - & - \\
\hline Y & - & - & - & - & - & - & - & - & - & - & - & - & - & - & - & - & - & - & - & - & - & - & - & - & - & - \\
\hline Z & - & - & - & - & - & $+1-$ & $+1-$ & $+1-$ & - & - & - & - & - & - & - & - & - & - & - & - & - & - & - & - & - & $+1-$ \\
\hline
\end{tabular}

${ }^{a}$ All $V$. dahliae isolates have been matched to a one-letter code (A to $Z$; Table 1) for the sake of data presentation.

'The vigour of complementation reactions is symbolized as '+' (for 'strong' reactions leading to dense prototrophic mycelial growth, usually accompanied by extended microsclerotia production), ' + - -' (for 'weak' reactions characterized by slight mycelial growth or only limited microsclerotia production), or '-' (the absence of macroscopically visible mycelial growth).

${ }^{C}$ The full VCG assignment of isolates $L$ and $M$, which are known to complement tester isolates of more than one VCG subgroups, is $2 B$ (2A) and $2 A B$ $(1,4 \mathrm{~A})$, respectively (secondary VCG affinities are provided in brackets; Table 1). HSI: heterokaryon self-incompatible.

dIsolate D - V661I (328 v-1) - was originally reported as heterokaryon self-incompatible (HSI), but vegetatively compatible with isolates of VCG 1B (Collado-Romero et al. 2006). 
Although these mutants were recovered by both methods, they were more abundant among the UVinduced and significantly more frequent among the chlorate-resistant mutants of $V$. dahliae isolates PCW and CA26 (21.4 and 37.5\%, respectively). Similarly, ten mutants unable to grow normally on any of the three nitrogen sources, but growing on PDA and on MM with ammonium tartrate as the sole nitrogen source, were identified mainly among the UV-induced mutants. Finally, no nit mutants were recovered from a single isolate (TO-0), as it appeared inherently resistant to chlorate at the concentration that was used in this work.

To examine the genetic stability of nit mutants, the reversion rates of 46 randomly selected mutants were determined (Table S2). Among these mutants, stability varied remarkably in a very wide range of reversion rates from approximately $10^{-3}$ to $10^{-8}$. Notably, the reversion rates were associated with individual isolates in an isolate-specific manner, with intra-isolate variation among independent mutants never exceeding two orders of magnitude (Table S2). On the other hand, no correlation with VCGs, method of mutants' production or mutant class was detected.

\section{A high-throughput procedure for complementation testing of nit mutants}

The rapid and high-throughput method developed here for the classification of $V$. dahliae isolates into VCGs involved standard 96-well microplates containing liquid $M M$, in which nit mutants were paired in all possible combinations, in four to eight repetitions of each pairing. With the exception of isolate TO-0, from which no nit mutants were recovered, 26 isolates of all VCGs were tested (Table 1; an example is illustrated in Fig. 1). Results were clear-cut and highly reproducible (averaging $97.5 \%$ between repetitions of each pairing) 10 days after inoculation, and they are summarized in Table 2. After recording results in microplate wells, the mycelial mats of 24 randomly selected pairings were transferred to the surface of solid MM, where they all retained slow growth ability, while their conidia could not grow on MM, suggesting that they were produced by actual heterokaryons rather than revertants. The results from the new method were in excellent agreement with the traditional complementation tests (an overall congruence value of $96.0 \%$ was recorded). Furthermore, the effect of nit mutants' stability on complementation testing was studied. Notably, when mutants with reversion rates higher than $10^{-6}$ were used (Table
S2), false-positive results were frequently obtained with both methods. This was presumably due to reversion to prototrophy and subsequent growth on MM, underlining the need for careful selection of nit mutants prior to complementation testing with either method.

Except for VCGs 1A and 6, which never exhibited cross-reactions with other VCGs, several 'bridging' reactions were recorded with both methods between all other VCGs and their subgroups, including pairings with some of the widely used international VCG tester isolates, for example $\mathrm{PH}, 115,70-21, \mathrm{BB}$ and S39 (Table 2). The extent of prototrophic growth in these cross-reactions ranged from limited mycelial development ('weak' reactions) to the production of small dots of microsclerotia at the contact zone (marginal interactions) (some examples are shown in Fig. 2). Five of the tested $V$. dahliae isolates that had been previously assigned to VCGs (Table 1), that is V607I and V661I (originally characterized as VCG $1 \mathrm{~B}$ and $1 \mathrm{~B}$ HSI, respectively), V830 and Dvd-E6 (VCG 4A), and Cf.162 (VCG 6), generally failed to complement other isolates from the collection tested. Complementary self-pairings of these isolates were also consistently unsuccessful, even though all of their available nit mutants were tested in several repetitions and all possible combinations. These isolates were, therefore, characterized here as heterokaryon self-incompatible (HSI).

\section{Heterokaryon analysis}

To investigate the genetic differences between 'strong' and 'weak' complementation interactions, ten presumed heterokaryons from each of the 'strong' and 'weak' types were transferred to solid MM. When sterile cellophane sheets were interposed between the two inocula of each pairing, no growth was observed, suggesting that complementation was not due to crossfeeding (syntrophism). It was also observed that heterokaryons deriving from originally 'weak' interactions had generally slower growth rates than those from 'strong' reactions, were highly irregular in the shape of the colony and pigment distribution, occasionally produced fast-growing sectors and were frequently predominated by one of the parental types (most often the nitM type; data not shown) after one to three subculturings on MM (examples are shown in Fig. 2). In contrast, most heterokaryons from 'strong' reactions were stable in culture after at least five subculturings.

For further analyses, three heterokaryons of each of the 'strong' and 'weak' types - including heterokaryons between different VCGs or subgroups - were 
(a)
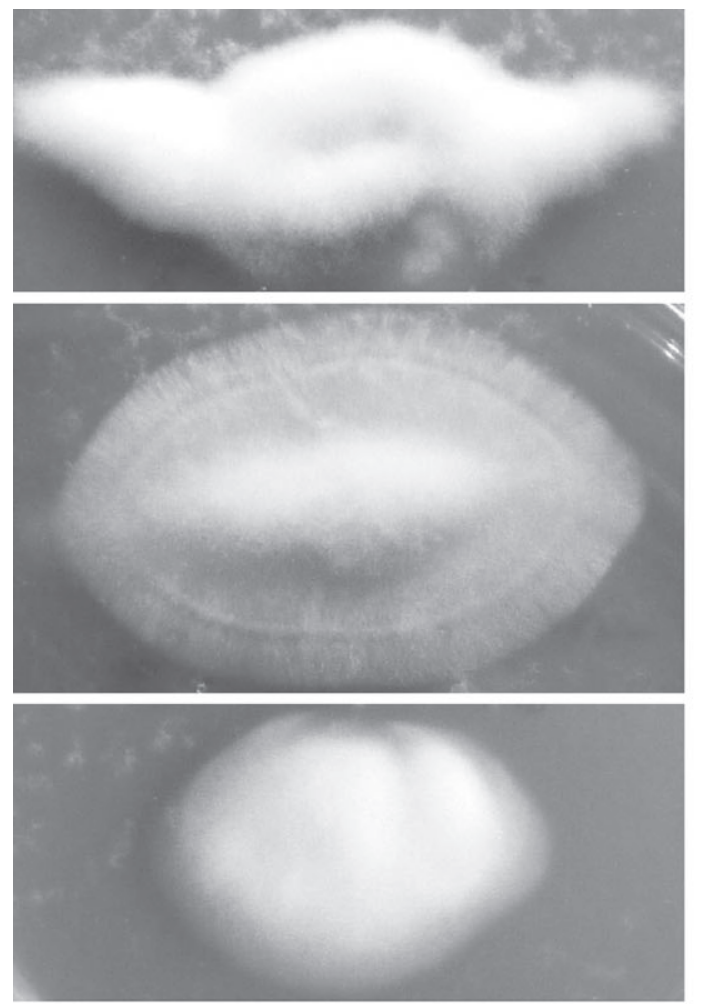

(b)
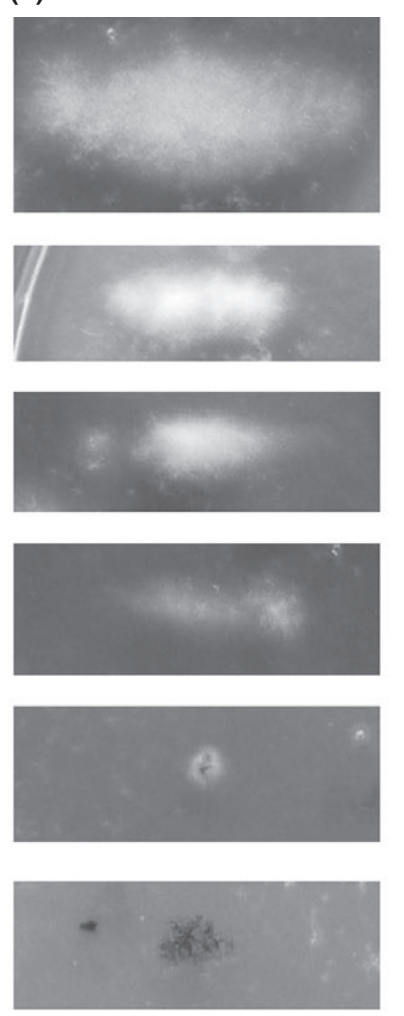

(c)

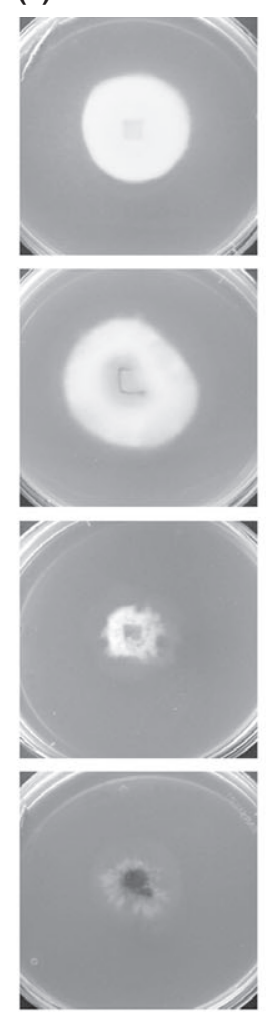

(d)

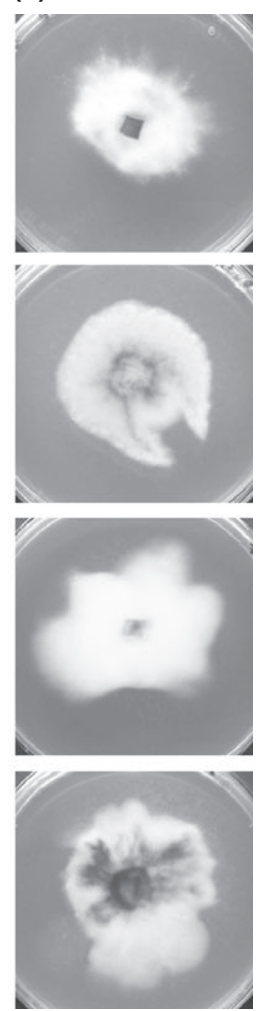

Fig. 2 Examples of morphological types of heterokaryons formed by complementary nit mutants of Verticillium dahliae isolates on solid MM. (a) 'Strong' complementation, characterized by compact colonies with dense aerial mycelium and high growth rate. (b) 'Weak' and 'marginal' complementation, ranging from slow prototrophic growth with sparse aerial mycelium, to isolated tufts of hyphae and microsclerotia or small dots of microsclerotia; these weak interactions were often non-reproducible between independent repetitions of the same pairing. Photographs of all heterokaryons in panels (a) and (b) were taken 4 weeks after inoculation of the pairings and were scaled proportionately to each other. (c) Heterokaryons derived from both 'strong' (the two upper photographs) and 'weak' (the two lower photographs) interactions could be perpetuated on MM with subculturings from the central heterokaryotic area, even though the latter heterokaryons grew at considerably slower rates and their margins were more irregular (photographs taken 2 weeks after transfer). (d) Four-week-old heterokaryotic colonies on MM with typical heterokaryon characteristics, including highly irregular growth (concerning both shape of colony and pigment distribution), and the emergence of fast-growing sectors.

Table 3 Conidial and hyphal tip analyses of Verticillium dahliae heterokaryons

\begin{tabular}{|c|c|c|c|c|c|}
\hline \multicolumn{2}{|c|}{ Heterokaryon components } & \multirow{2}{*}{$\begin{array}{l}\text { Nuclear A:B ratio } \\
\text { among conidia } \\
\text { (No. tested) }\end{array}$} & \multicolumn{2}{|c|}{$\begin{array}{l}\text { No. of hyphal tips } \\
\text { of nuclear type }\end{array}$} & \multirow{2}{*}{$\begin{array}{l}\text { Mean colony } \\
\text { diameter } \\
(\mathrm{mm})^{\mathrm{a}}\end{array}$} \\
\hline A (nit1) & B (nitM) & & A & B & \\
\hline \multicolumn{6}{|c|}{ Heterokaryons derived from initially ‘strong' reactions } \\
\hline Ls.17.10S (VCG 2B) & Ls.17.15S (VCG 2B) & 1.0: $2.9(525)$ & 56 & 24 & 26 \\
\hline T9.8U (VCG 1A) & V44.15S (VCG 1A) & $1.0: 3.8(489)$ & 24 & 76 & 24 \\
\hline 70-21.7U (VCG 3) & PCW.20U (VCG 3) & 1.9: $1.0(505)$ & 58 & 42 & 20 \\
\hline \multicolumn{6}{|c|}{ Heterokaryons derived from initially ‘weak' reactions } \\
\hline 115.1S (VCG 2B) & Cf38.42S (VCG 2B) & 1.0: $5.4(516)$ & 38 & 52 & 17 \\
\hline V320I.5U (VCG 2A) & Ls.17.15S (VCG 2B) & 1.0: $8.6(435)$ & 6 & 74 & 11 \\
\hline 530-1.23S (VCG 2AB) & S39.45S (VCG 4B) & 3.4: $1.0(412)$ & 41 & 59 & 15 \\
\hline
\end{tabular}

${ }^{a}$ Mean heterokaryon diameter after incubation for 8 days at $24^{\circ} \mathrm{C}$, in the dark.

randomly selected (Table 3). Mycelial agar block transfers from the centre of all six heterokaryons always grew on MM, giving rise to characteristic compact colonies with a wide outer margin of thin mycelium, which resembled the growth of nit mutants (Fig. 2), and slower growth rates than the 
parental nit mutants on appropriately supplemented MM. Conidia of 4-week-old heterokaryons invariably retained auxotrophy when spread on MM, and when analysed for their genotypes, they showed ratios ranging from approximately $1: 1$ to $1: 9$, most often being skewed towards the nitM type. These ratios were more disproportionate for 'weak' rather than for 'strong' heterokaryons (Table 3). When 0.5-mm-wide hyphal tips from the margins of heterokaryons were transferred to MM, they were homokaryotic for either one or the other nit marker, with one predominating (Table 3).

\section{Discussion}

Although nit mutants-based VCG complementation studies have been widely used for the characterization of $V$. dahliae populations for more than two decades, the available methods are insufficient for use in largescale analyses (Leslie 1993). In this work, UV-mutagenesis was applied for the generation of nit mutants, and these were inoculated into liquid microcultures for the rapid, consistent and high-throughput VCG assignment of $V$. dahliae isolates. With our optimized protocols, we demonstrate that both steps can be reliably accomplished in considerably less time than with the traditional procedures and used for the simultaneous testing of hundreds of independent pairings in each experiment, without the need for special equipment, extra storage space or additional applicant's expertise. Notably, under the mutagenesis conditions that were used, the phenotype distribution, genetic stability and complementation behaviour of UVinduced nit mutants were essentially identical with those deriving from spontaneous chlorate-resistant sectors. These features, together with the high reproducibility and excellent congruence with the traditional VCG classification procedures, render the new method a highly suitable candidate for the large-scale VCG profiling of extended populations of $V$. dahliae and probably other fungi.

Great variability in the extent of prototrophic growth between different pairings, or even between independent nit mutants of the same pair of isolates, has been observed in most VCG classification studies (Joaquim and Rowe 1990; Korolev et al. 2000; Jiménez-Díaz et al. 2006; Göre 2009). To avoid misinterpretations, 'strong' reactions, resembling the wild type of the fungus in the amount of prototrophic growth achieved, were almost exclusively used for the classification of isolates into the VCG system. However, 'weaker' reactions accounting for the crossVCG (or 'bridging') interactions between different
VCGs and their subgroups were also frequently reported, thus underlining the complexity of the genetics of the VCG system (Joaquim and Rowe 1990, 1991; Elena and Paplomatas 1998; Hiemstra and Rataj-Guranowska 2003; El-Bebany et al. 2013; Papaioannou et al. 2013b). In this study, we performed a genetic analysis and comparison of the two types of complementation interactions, and we demonstrated that (i) 'weak' reactions within and between VCGs can be due to actual heterokaryosis, similar to the 'strong' responses, and (ii) heterokaryons deriving from 'weak' interactions are unstable, with an uneven distribution of genetically different nuclei tending to segregate into novel homokaryotic mycelia. According to the general assumption underlying VCG analysis, that is that each VCG consists of genetically similar isolates with identical het genotypes, heterokaryon compatibility has been predicted to be restricted to the members of the same VCG; different VCGs, on the other hand, have been regarded as incompatible groupings of isolates due to differences at one or more het loci (Leslie 1993; Katan 2000). Notwithstanding this hypothesis, the 'weak' inter-VCG heterokaryosis observed in this and other studies implies the absence of strict genetic barriers between certain $V$. dahliae VCGs. This may involve serious implications for the interpretation of traditional VCG classification data, especially if actual genetic recombination between different VCGs is demonstrated in future studies. Based on the available data, an attractive hypothesis would be that a spectrum between highly stable and more unstable heterokaryosis exists in $V$. dahliae instead of clear-cut compatibility or incompatibility. Alternatively, the unstable 'weak' heterokaryosis might merely be an artefact of the traditional VCG assignment method that involves testing compatibility under forced conditions. These possibilities have to be addressed in future genetic investigations for the clarification of the situation.

Until a more comprehensive understanding of the het gene system underlying compatibility of $V$. dahliae is attained, population analyses are used to shed some light on the genetic relationships within and between VCGs. Several recent studies have challenged the traditional principle of VCG clonality, showing altogether that (i) not all VCGs or their subgroups correspond to clonal lineages; for example, VCG $2 \mathrm{~B}$ is heterogeneous and polyphyletic, comprising isolates of more than one distinct molecular types that are grouped with different VCGs, and (ii) main VCGs 2 and 4 consist of genetically unrelated subgroups which are similar to subgroups of other VCGs (Collado-Romero et al. 2008; Martin 2010; Papaioannou 
et al. 2013a,b; Jiménez-Gasco et al. 2014). These findings may be possibly related to observations of 'weak' heterokaryosis made previously and in this work. Marked heterogeneity has been observed in the compatibility behaviour of isolates of the polyphyletic VCG 2B, with certain representatives complementing several but not all other VCG 2B members, and various others exhibiting usually weak 'bridging' ability with other VCGs (Jiménez-Díaz et al. 2006; Papaioannou et al. 2013b; present work). Furthermore, 'bridging' isolates between most of the known VCG subgroups have been frequently detected (Joaquim and Rowe 1990, 1991; Elena and Paplomatas 1998; Hiemstra and Rataj-Guranowska 2003; El-Bebany et al. 2013; Papaioannou et al. 2013b; present work), including, for example, the pair of VCG subgroups $2 \mathrm{~A}$ and $4 \mathrm{~B}$, which have been shown to be indistinguishable at several molecular characteristics (Martin 2010; Papaioannou et al. 2013a,b; Jiménez-Gasco et al. 2014). The hypothesis that the usually 'weak' heterokaryons formed between these 'bridging' pairs of isolates may permit exchange of genetic material between VCGs could explain some of the previously unexpected phylogenetic affinities between certain VCG subgroups, for example $2 \mathrm{~B}$ and $4 \mathrm{~A}$ of the example mentioned above. In addition, the observation in the present study that VCGs 1 and 6 never exhibited cross-reactions with other VCGs is also in concordance with phylogenetic data that show them to be rather clonal entities (Collado-Romero et al. 2008; Martin 2010; Papaioannou et al. 2013a; JiménezGasco et al. 2014) (considering the revisited classification of VCG 6 isolates; Papaioannou et al. 2014). The possibility of parasexual genetic recombination between VCGs is further supported by the previous demonstration that the exchange of genetic material between incompatible isolates can be induced experimentally in Verticillium through 'weak' heterokaryosis under appropriately forced conditions (Typas 1983).

Although the sexual state of $V$. dahliae has never been found, mating-type idiomorphs are present in this species (Usami et al. 2009), and population analyses have revealed putative signs of recombination (Atallah et al. 2010; Inderbitzin et al. 2011). Based on these findings, it cannot be ruled out that part of the observed variation and the deviation from clonality according to the VCG system may result from rare or even ancient sexual recombination events. Although parasexuality has been experimentally established in Verticillium (Puhalla and Mayfield 1974; Typas and Heale 1978), its importance in natural populations of fungi has never been unequivocally demonstrated to date. Therefore, sexual recombination might explain some of the observed patterns of variation in $V$. dahliae, and future research in this direction is especially welcome.

Another interesting finding of this work is the variety of chlorate-resistant mutants, especially after UVtreatment. Mutants unable to grow on all nitrogen sources except for ammonium may be defective in the major nitrogen regulatory gene are $A$ (Lenouvel et al. 2001), while additional rare phenotypes were also recorded. Interestingly, a remarkably broad and isolate-specific range of reversion rates for individual nit mutants was detected, and an impact of their stability on the results of complementation tests was demonstrated. These data underline the need for careful selection of appropriate nit mutants prior to complementation studies. Furthermore, we found evidence for a selective advantage of the nitM type over nitl mutations in $V$. dahliae heterokaryons. The possible relevance of this genetic imbalance to the reliability of complementation tests merits further examination. Finally, it was observed that five of the tested $V$. dahliae isolates that had previously been assigned to VCGs, appeared consistently heterokaryon selfincompatible in this study and unable to complement any other isolate of the collection. This phenomenon could be explained by the hypothesis of Jacobson and Gordon (1990), who tried to explain self-incompatibility in Fusarium oxysporum as a mutant artefact that arises in fungi maintained for long periods in artificial culture, although further study is necessary for a solid conclusion.

In conclusion, our work provides (i) an optimized method for the high-throughput VCG classification of $V$. dahliae isolates using nit mutants on a large scale and (ii) a genetic analysis of heterokaryons between nit mutants of $V$. dahliae isolates, which implies the absence of strict genetic barriers between VCGs. The results suggest that a systematic re-evaluation of vegetative incompatibility in Verticillium and its biological significance is essential for the unravelling of the population biology of the fungus. Novel methods suitable for large-scale application, such as the one provided here, are hopefully expected to facilitate this endeavour.

\section{Acknowledgements}

We would like to thank all individuals listed in the footnote of Table 1 for providing fungal isolates used in this study. I. A. Papaioannou and this research have been cofinanced by the European Union (European Social Fund - ESF) and Greek national funds through the Operational Program 'Education and Lifelong Learning' of the National Strategic Reference 
Framework (NSRF) - Research Funding Program: Heracleitus II, investing in knowledge society through the European Social Fund.

\section{References}

Anderson JB, Kohn LM. (1995) Clonality in soilborne, plant-pathogenic fungi. Annu Rev Phytopathol 33:369391.

Atallah ZK, Maruthachalam K, du Toit L, Koike ST, Davis RM, Klosterman SJ, Hayes RJ, Subbarao KV. (2010) Population analyses of the vascular plant pathogen Verticillium dahliae detect recombination and transcontinental gene flow. Fungal Genet Biol 47:416-422.

Bhat RG, Smith RF, Koike ST, Wu BM, Subbarao KV. (2003) Characterization of Verticillium dahliae isolates and wilt epidemics of pepper. Plant Dis 87:789-797.

Collado-Romero M, Mercado-Blanco J, Olivares-García C, Valverde-Corredor A, Jiménez-Díaz RM. (2006) Molecular variability within and among Verticillium dahliae vegetative compatibility groups determined by fluorescent amplified fragment length polymorphism and polymerase chain reaction markers. Phytopathology 96:485495.

Collado-Romero M, Mercado-Blanco J, Olivares-García C, Jiménez-Díaz RM. (2008) Phylogenetic analysis of Verticillium dahliae vegetative compatibility groups. Phytopathology 98:1019-1028.

Collins A, Mercado-Blanco J, Jiménez-Díaz RM, Olivares C, Clewes E, Barbara DJ. (2005) Correlation of molecular markers and biological properties in Verticillium dahliae and the possible origins of some isolates. Plant Pathol 54:549-557.

Correll JC, Klittich CJR, Leslie JF. (1987) Nitrate nonutilizing mutants of Fusarium oxysporum and their use in vegetative compatibility tests. Phytopathology 77:16401646.

Daayf F, Nicole M, Geiger J-P. (1995) Differentiation of Verticillium dahliae populations on the basis of vegetative compatibility and pathogenicity on cotton. Eur J Plant Pathol 101:69-79.

Davey JW, Hohenlohe PA, Etter PD, Boone JQ, Catchen JM, Blaxter ML. (2011) Genome-wide genetic marker discovery and genotyping using next-generation sequencing. Nat Rev Genet 12:499-510.

Dobinson KF, Patterson NA, White GJ, Grant S. (1998) DNA fingerprinting and vegetative compatibility analysis indicate multiple origins for Verticillium dahliae race 2 tomato isolates from Ontario, Canada. Mycol Res 102:1089-1095.

Dung JKS, Peever TL, Johnson DA. (2013) Verticillium dahliae populations from mint and potato are genetically divergent with predominant haplotypes. Phytopathology 103:445-459.
El-Bebany AF, Alkher H, Adam LR, Daayf F. (2013) Vegetative compatibility of Verticillium dahliae isolates from potato and sunflower using nitrate non-utilizing (nit) mutants and PCR-based approaches. Can J Plant Pathol 35:1-9.

Elena K, Paplomatas EJ. (1998) Vegetative compatibility groups within Verticillium dahliae isolates from different hosts in Greece. Plant Pathol 47:635-640.

Göre ME. (2009) Vegetative compatibility and pathogenicity of Verticillium dahliae isolates from chrysanthemum in Turkey. Phytoparasitica 37:87-94.

Gurung S, Short DPG, Atallah ZK, Subbarao KV. (2014) Clonal expansion of Verticillium dahliae in lettuce. Phytopathology 104:641-649.

Hiemstra JA, Rataj-Guranowska M. (2003) Vegetative compatibility groups in Verticillium dahliae isolates from the Netherlands as compared to VCG diversity in Europe and in the USA. Eur J Plant Pathol 109:827-839.

Hutchison EA, Glass NL. (2012) Programmed cell death and heterokaryon incompatibility in filamentous fungi. In: Witzany G (ed) Biocommunication of Fungi. Dordrecht, Netherlands, Springer Science+Business Media, pp 115-138.

Iglesias-Garcia AM, Villarroel-Zeballos MI, Feng C, du Toit LJ, Correll JC. (2013) Pathogenicity, virulence, and vegetative compatibility grouping of Verticillium isolates from spinach seed. Plant Dis 97:1457-1469.

Inderbitzin P, Davis RM, Bostock RM, Subbarao KV. (2011) The ascomycete Verticillium longisporum is a hybrid and a plant pathogen with an expanded host range. PLoS ONE 6:e18260.

Jacobson DJ, Gordon TR. (1990) Further investigations of vegetative compatibility within Fusarium oxysporum $\mathrm{f}$. sp. melonis. Can J Bot 68:1245-1248.

Jiménez-Díaz RM, Mercado-Blanco J, Olivares-García C, Collado-Romero M, Bejarano-Alcázar J, Rodríguez-Jurado D, Giménez-Jaime A, García-Jiménez J, Armengol J. (2006) Genetic and virulence diversity in Verticillium dahliae populations infecting artichoke in eastern-central Spain. Phytopathology 96:288-298.

Jiménez-Gasco MM, Malcolm GM, Berbegal M, Armengol J, Jiménez-Díaz RM. (2014) Complex molecular relationship between Vegetative Compatibility Groups in Verticillium dahliae: VCGs do not always align with clonal lineages. Phytopathology 104:650-659.

Joaquim TR, Rowe RC. (1990) Reassessment of vegetative compatibility relationships among strains of Verticillium dahliae using nitrate-nonutilizing mutants. Phytopathology 80:1160-1166.

Joaquim TR, Rowe RC. (1991) Vegetative compatibility and virulence of strains of Verticillium dahliae from soil and potato plants. Phytopathology 81:552-558.

de Jonge R, Bolton MD, Kombrink A, van den Berg GCM, Yadeta KA, Thomma BPHJ. (2013) Extensive 
chromosomal reshuffling drives evolution of virulence in an asexual pathogen. Genome Res 23:1271-1282.

Katan T. (2000) Vegetative compatibility in populations of Verticillium - an overview. In: Tjamos EC, Rowe RC, Heale JB, Fravel DR. (eds) Advances in Verticillium: research and disease management. Proceedings of the 7th International Verticillium Symposium. St Paul, MN, USA, APS Press, pp 69-86.

Klittich CJR, Leslie JF. (1988) Multiwell plates for complementation tests of Fusarium. Fungal Genet Newsl 35:2122.

Klosterman SJ, Atallah ZK, Vallad GE, Subbarao KV. (2009) Diversity, pathogenicity and management of Verticillium species. Annu Rev Phytopathol 47:39-62.

Korolev N, Katan T. (1997) Improved medium for selecting nitrate-nonutilizing (nit) mutants of Verticillium dahliae. Phytopathology 87:1067-1070.

Korolev N, Katan J, Katan T. (2000) Vegetative compatibility groups of Verticillium dahliae in Israel: their distribution and association with pathogenicity.

Phytopathology 90:529-536.

Lenouvel F, Fraissinet-Tachet L, van de Vondervoort P, Visser J. (2001) Isolation of UV-induced mutations in the are A nitrogen regulatory gene of Aspergillus niger, and construction of a disruption mutant. Mol Genet Genomics 266:42-47.

Leslie JF. (1993) Fungal vegetative compatibility. Annu Rev Phytopathol 31:127-150.

López-Escudero FJ, Mercado-Blanco J. (2011) Verticillium wilt of olive: a case study to implement an integrated strategy to control a soil-borne pathogen. Plant Soil 344:1-50.

Martin FN. (2010) Mitochondrial haplotype analysis as a tool for differentiating isolates of Verticillium dahliae. Phytopathology 100:1231-1239.

Papaioannou IA, Dimopoulou CD, Typas MA. (2013a) Structural and phylogenetic analysis of the rDNA intergenic spacer region of Verticillium dahliae. FEMS Microbiol Lett 347:23-32.

Papaioannou IA, Ligoxigakis EK, Vakalounakis DJ, Markakis EA, Typas MA. (2013b) Phytopathogenic, morphological, genetic and molecular characterization of a Verticillium dahliae population from Crete, Greece. Eur J Plant Pathol 136:577-596.

Papaioannou IA, Dimopoulou CD, Typas MA. (2014) "Cryptic" group-I introns in the nuclear SSU-rRNA gene of Verticillium dahliae. Curr Genet 60:135-148.
Puhalla JE, Hummel M. (1983) Vegetative compatibility groups within Verticillium dahliae. Phytopathology 73:1305-1308.

Puhalla JE, Mayfield JE. (1974) The mechanisms of heterokaryotic growth in Verticillium dahliae. Genetics 76:411-422.

Puhalla JE, Spieth PT. (1983) Heterokaryosis in Fusarium moniliforme. Exp Mycol 7:328-335.

Strausbaugh CA, Schroth MN, Weinhold AR, Hancock JG. (1992) Assessment of vegetative compatibility of Verticillium dahliae tester strains and isolates from California potatoes. Phytopathology 82:61-68.

Tsror (Lahkim) L, Hazanovsky M, Mordechi-Lebiush S, Sivan S. (2001) Aggressiveness of Verticillium dahliae isolates from different vegetative compatibility groups to potato and tomato. Plant Pathol 50:477-482.

Typas MA. (1983) Heterokaryon incompatibility and interspecific hybridization between Verticillium albo-atrum and Verticillium dahliae following protoplast fusion and microinjection. J Gen Microbiol 129:30433056.

Typas MA, Heale JB. (1976) Heterokaryosis and the role of cytoplasmic inheritance in dark resting structure formation in Verticillium spp. Mol Gen Genet 146:17-26.

Typas MA, Heale JB. (1978) Heterozygous diploid analyses via the parasexual cycle and a cytoplasmic pattern of inheritance in Verticillium species. Genet Res 31:131144.

Usami T, Itoh M, Amemiya Y. (2009) Asexual fungus Verticillium dahliae is potentially heterothallic. J Gen Plant Pathol 75:422-427.

Zeise K, von Tiedemann A. (2001) Morphological and physiological differentiation among vegetative compatibility groups of Verticillium dahliae in relation to $\mathrm{V}$. longisporum. J Phytopathol 149:469-475.

\section{Supporting Information}

Additional Supporting Information may be found in the online version of this article:

Table S1. Nit mutants of Verticillium dahliae isolates that were produced and characterized in this work.

Table S2. Reversion rates of 46 nit mutants of Verticillium dahliae isolates produced by either UV-induction or selection of spontaneous chlorate-resistant sectors. 